\title{
Analyse Performance Of Fractional FOURIER TRANSFORM ON TIMING AND CARRIER FREQUENCY OFFSETS ESTIMATION
}

\author{
Cherif Rezgui \\ Communication Systems Lab, National School of Engineers of Tunis (ENIT), Tunisia
}

\begin{abstract}
This paper deals with the performance of the use of fractional Fourier transform (FRFT) instead of conventional Fourier transform (FFT) in either symbol timing offset (STO) and carrier frequency offset (CFO) estimation. Orthogonal frequency division multiplexing is widely used in many systems due to advantages of theses technique compared with mono-carrier systems. In spite of his advantages, OFDM presents drawbacks such as sensitivity to timing and frequency offsets. Many techniques are used in the literature to estimate these two parameters in order to compensate them (synchronization task). These techniques used conventional Fourier transform. In this paper, we are interested in estimating STO and CFO using fractional Fourier transform. Monte Carlo simulation demonstrates the performance of the use of FRFT instead of FFT.
\end{abstract}

\section{KEYWORDS}

FRFT, STO, CFO, CAZAC sequences, MSE, SNR

\section{INTRODUCTION}

The principle of OFDM is to divide, on a wide number of subcarriers, the digital signal that is to be transmitted. In wireless systems, transmitted data reaches to receivers after transit through a radio channel. For conventional coherent receivers, the effect of channel on transmitted signal must be estimated to recover transmitted data.

The signal is spread in frequency, time, and angle; therefore, the received signal suffers from the impact of these spreads on transmitted signal. In spite of many advantages of OFDM compared to single carrier systems, it has major drawbacks such carrier frequency and symbol timing offsets. So, estimating these two parameters (STO and CFO) is very important. Timing and carrier frequency synchronizations are the major task of OFDM receiver.

Various researches had been proposed to estimate CFO [2-6]...Other techniques are used in the literature to estimate STO [7-11]... These techniques, for both STO and CFO estimation, use conventional Fourier transform (FFT). This article is about estimating CFO and STO for known method with the use of fractional Fourier transform (FRFT) to illustrate the importance of FRFT compared to FFT.

DOI : 10.5121/ijwmn.2016.8201 
International Journal of Wireless \& Mobile Networks (IJWMN) Vol. 8, No. 2, April 2016

The remainder of this paper is organized as follows. In section 2, we introduce OFDM signal and the effect of CFO. Section 3 gives the effect of STO. Then, we present Fractional Fourier Transform in section 4. Thereafter, Section 5 shows the proposed method and results of simulations between the use of FFT and FRFT in terms of MSE. Conclusion is given in the last section.

\section{OFDM BASIC AND CFO}

OFDM signal is the sum of many independent signals modulated onto sub-channels of equal bandwidth. Let us define $\mathrm{N}$ symbols in OFDM as $\left\{X_{n}, n=0,1, \ldots, N\right\}$ The complex baseband representation of a multicarrier signal consisting of $\mathrm{N}$ subcarriers is given by :

$$
x_{l}(t)=\frac{1}{\sqrt{N}} \sum_{k=0}^{N-1} X_{l}(k) e^{j 2 \pi \Delta f k t} ; 0 \leq t<N T
$$

Where $\mathrm{j}=\sqrt{ }-1, \Delta \mathrm{f}$ is the subcarrier spacing, 1 the $1^{\text {th }}$ OFDM symbol and NT denotes the useful data block period. The subcarriers in OFDM system are supposed to be orthogonal to each other. In order to demodulate an OFDM symbol correctly at the receiver using N point DFT, it is very much required to take exact samples of transmitted OFDM symbol. The signal $\mathrm{x}(\mathrm{t})$ is modulated by a carrier frequency $\mathrm{f}_{0}$ in the transmitter, then demodulated at the reception at the same frequency. This is the ideal case where the frequency of modulation and demodulation are identical. But this case does not happen in practice because these frequencies are derived from different oscillators (at the transmitter and receiver) which each have defects. Note that the following assumption has been made implicitly in the baseband model. Let $\mathrm{f}_{\mathrm{c}}{ }^{\mathrm{t}}$ and $\mathrm{f}_{\mathrm{c}}{ }^{\mathrm{r}}$ denote the carrier frequencies in the transmitter and receiver, respectively. Let $\mathrm{f}_{\text {offset }}$ denote their difference (i.e., $f_{\text {offset }}=f_{c}{ }^{t}-f_{c}^{r}$ ). Meanwhile, Doppler frequency $f_{d}$ is determined by the carrier frequency $f_{c}{ }^{t}$ and the velocity $\mathrm{v}$ of the terminal (receiver) as:

$$
f_{d}=\frac{v \cdot f_{c}^{t}}{c}
$$

The normalized carrier frequency offset $(\mathrm{CFO}), \varepsilon$, is defined as:

$$
\varepsilon=\frac{f_{\text {offset }}}{\Delta f}
$$

The time-domain received signal, with the presence only of CFO (no Phase Noise), can be written as:

$$
y_{l}(n)=\frac{1}{N} \sum_{k=0}^{N-1} H(k) X_{l}(k) e^{j 2 \pi(k+\varepsilon) \frac{n}{N}}+z_{l}(n)
$$

where $X_{1}(k)$ and $H_{1}(k)$ denote the $k^{\text {th }}$ subcarrier frequency components of the $1^{\text {th }}$ transmitted symbol and channel frequency response in the frequency domain respectively, and $z_{1}(n)$ noise in the time domain.

The table 1 presents impact of CFO in time and frequency domain signal. The frequency shift of $-\varepsilon$ in the frequency-domain signal $\mathrm{X}(\mathrm{k})$ subjects to the CFO of $\varepsilon$ and leads to an inter-carrier interference (ICI), which means a subcarrier frequency component is affected by other subcarrier frequency components. It is therefore essential to estimate it in order to resolve problems such as Inter-Carrier Interference. 
International Journal of Wireless \& Mobile Networks (IJWMN) Vol. 8, No. 2, April 2016

Table 1: The effect of CFO on the received signal, [1]

\begin{tabular}{|l|l|l|}
\hline & Received signal & Effect of STO $\delta$ on the received signal \\
\hline Time-domain signal & $\mathrm{y}(\mathrm{n})$ & $\mathrm{e}^{\mathrm{j} 2 \pi \mathrm{k} \varepsilon / \mathrm{N}} \mathrm{x}(\mathrm{n})$ \\
\hline Frequency-domain signal & $\mathrm{Y}(\mathrm{k})$ & $\mathrm{X}(\mathrm{k}-\varepsilon)$ \\
\hline
\end{tabular}

The most known methods that estimate CFO are Moose [2] and Classen [3] mehods. It was demonstrated in the literature that Classen method is better than the one done by Moose. That's why we are interested in estimating CFO using Classen method but the use of FRFT. The main idea of Classen is: pilot tones can be inserted in the frequency domain and transmitted in every OFDM symbol for CFO tracking. First, two OFDM symbols, $\mathrm{y}_{1}(\mathrm{n})$ and $\mathrm{y}_{1+\mathrm{D}}(\mathrm{n})$ are saved in the memory after synchronization. Then, the signals are transformed into $Y_{1}(K)^{N-1} k=0$ and $\quad Y_{1+D}(k)^{N-}$ $1_{\mathrm{k}=0}$ via FFT, from which pilot tones are extracted. After estimating CFO from pilot tones in the frequency domain, the signal is compensated with the estimated CFO in the time domain. In this process, two different estimation modes for $\mathrm{CFO}$ estimation are implemented: acquisition and tracking modes. In the acquisition mode, a large range of CFO including an integer CFO is estimated. In the tracking mode, only fine CFO is estimated. The integer CFO is estimated by:

$$
\hat{\varepsilon}_{a c q}=\frac{1}{2 \pi T_{s u b}} . \quad \max _{\varepsilon}\left\{\left|\sum_{j=0}^{L-1} Y_{l+D}(p(j), \varepsilon) Y_{l}^{*}(p(j), \varepsilon) X_{l+D}^{*}(p(j)) X_{l}(p(j))\right|\right\}
$$

where $L, p(j)$, and $X_{1}(p(j))$ denote the number of pilot tones, the location of the $j^{\text {th }}$ pilot tone, and the pilot tone located at $\mathrm{p}(\mathrm{j})$ in the frequency domain at the $1^{\text {th }}$ symbol period, respectively. $\mathrm{D}$ is an integer that represents the ratio of the OFDM symbol length to the length of a repetitive pattern.

\section{EFFECT OF STO}

In order to correctly demodulate an OFDM symbol at the receiver using $\mathrm{N}$ point DFT, it is very important to take exact samples of transmitted OFDM symbol. The correct starting point of DFT window is needed to preserve the orthogonality among the sub-carriers. OFDM systems present many advantages in comparison with single carrier system however all these advantages can be relevant only when the orthogonality among sub-carriers is kept. If one DFT window takes sample of two different OFDM symbol generating ICI and ISI. Table 2 shows the effect of timing offset in the received signal in time and frequency domain the effects of channel and noise are neglected for simplicity of exposition. The presence of STO in the time domain causes phase offset of $2 \pi \mathrm{kd} / \mathrm{N}$ in the frequency domain. This phase offset is proportional to the subcarrier index $\mathrm{k}$ as well as the STO $\delta$, Table 2 .

Table 2: The effect of STO on the received signal, [1]

\begin{tabular}{|l|l|l|}
\hline & Received signal & Effect of STO $\delta$ on the received signal \\
\hline Time-domain signal & $\mathrm{y}(\mathrm{n})$ & $\mathrm{x}(\mathrm{n}+\delta)$ \\
\hline Frequency-domain signal & $\mathrm{Y}(\mathrm{k})$ & $\mathrm{e}^{\mathrm{j} 2 \pi \mathrm{k}} / \mathrm{N} \mathrm{X}(\mathrm{k})$ \\
\hline
\end{tabular}

Four possible cases may occur, Figure 1. The first case occurs when there is no timing error (i.e. timing offset $\delta=0$ ). That is the case that estimated starting point of the OFDM symbol coincides with the exact time, preserving the orthogonality among sub-carriers, so the OFDM symbol can be perfectly left without any type of interference.The second case takes place when the estimated 
International Journal of Wireless \& Mobile Networks (IJWMN) Vol. 8, No. 2, April 2016

starting point of the OFDM symbol before the exact point, but after the end of the (displaced) channel response of the precedent OFDM symbol. In that case the $i^{\text {th }}$ symbol not overlapped with the preceding (i-1) ${ }^{\text {th }}$ OFDM symbol, that is, without incurring ISI by the preceding symbol in such cases. The third one is happen if the starting point of the OFDM symbol is estimated exist prior to the end of the (displaced) channel response of the previous OFDM symbol and thus the symbol synchronization is too soon to avoid the ISI. In that case the orthogonality between the components of subcarrier is destroyed by the ISI (the previous symbol) and furthermore, ICI occurs. The fourth case occurs if the starting point of the OFDM symbol is estimated just after the exact time. In this case, the samples for current FFT operation interval is consists of a part of the current OFDM symbol $\mathrm{x}_{1}(\mathrm{n})$ and a part of the following OFDM symbol $\mathrm{x}_{1+1}(\mathrm{n})$.

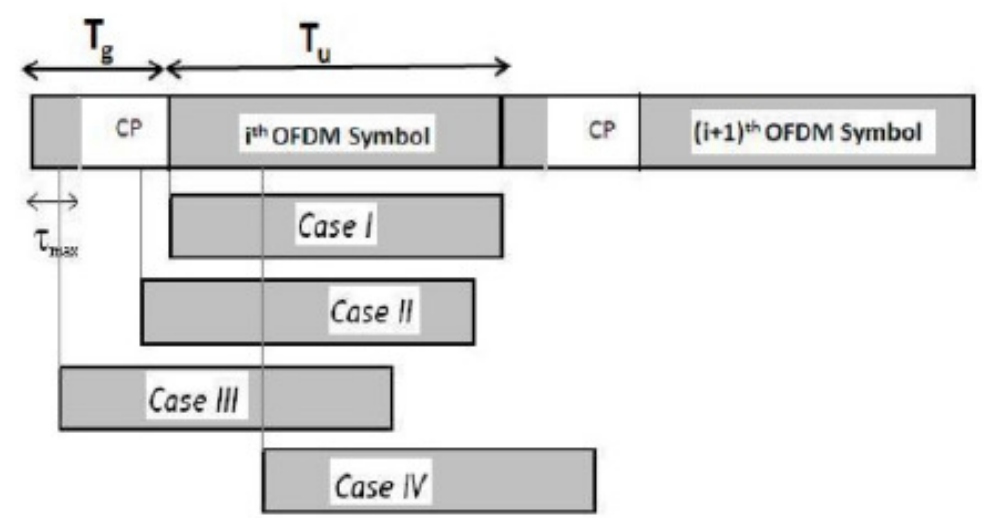

Figure 1: Four different cases symbol starting point subject to STO.

Many techniques in the literature are implemented using whether cyclic prefix or training sequence. As an example of estimating STO using cyclic prefix is done by Tourtier, P.J., Monnier, R., and Lopez, P. [7].

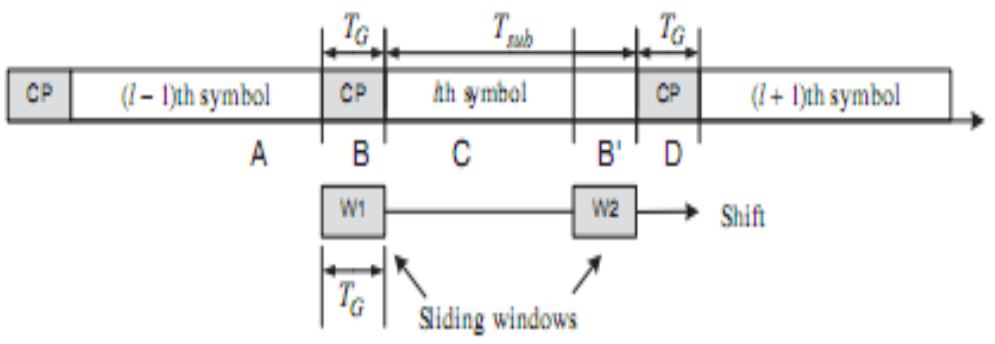

Figure 2: STO estimation technique using double sliding windows.

The estimated STO is done by:

$$
\hat{\delta}=\arg \min _{n}\left\{\sum_{i=\delta}^{N_{G^{-1}}+\delta}\left|y_{l}(n+i)-y_{l}(n+N+i)\right|\right\}
$$

Another technique used in [11]. It consists on minimizing the squared difference between a $\mathrm{N}_{\mathrm{G}}$ sample block (seized in window W1) and conjugate of another $\mathrm{N}_{\mathrm{G}}$ sample block (seized in window W2).

$$
\hat{\delta}=\arg \min _{n}\left\{\sum_{i=\delta}^{N_{G}-1+\delta}\left(\left|y_{l}(n+i)\right|-\left|y_{l}^{*}(n+N+i)\right|\right)^{2}\right\}
$$


International Journal of Wireless \& Mobile Networks (IJWMN) Vol. 8, No. 2, April 2016

Estimation techniques using training sequences are presented in Figure 3:

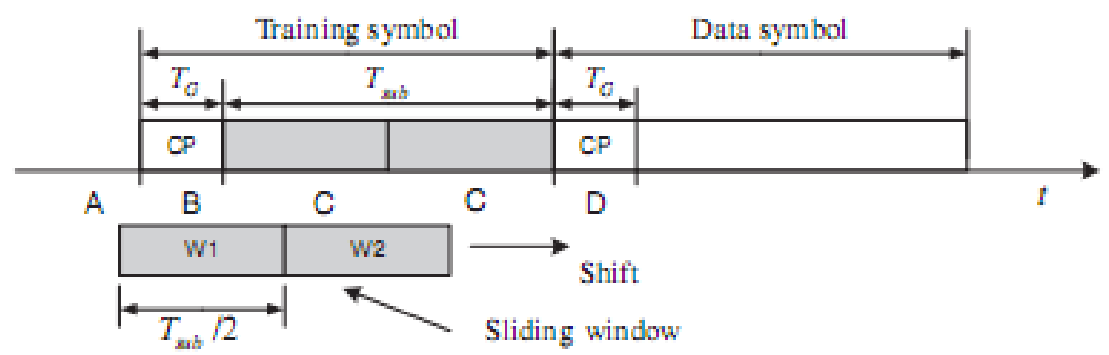

Figure 3: STO estimation using the repetitive training symbol, $($ period $=\mathrm{Tsub} / 2)$.

In this case, STO is estimated as, [9] and [10]:

$$
\delta=\arg \min _{n}\left\{\sum_{i=\delta}^{N_{G}-1+\delta}\left(\left|y l(n+i)-y_{l}^{*}(n+N / 2+i)\right|\right)^{2}\right\}
$$

In this paper, STO is estimated in frequency domain. As implied in second case, the received signal subject to STO suffers from a phase rotation, Figure 4:

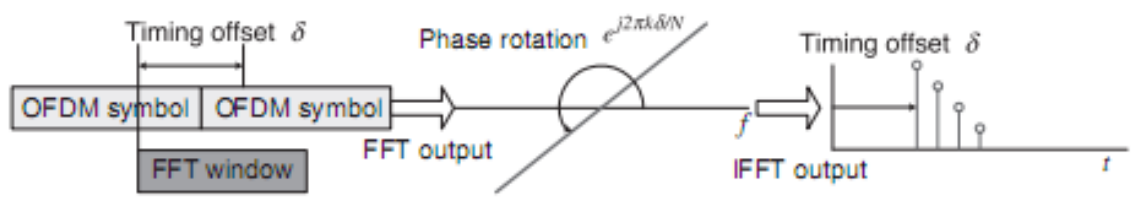

Figure 4 : Effect of STO in frequency domain

STO is estimated as:

Where ${ }^{y_{l}^{X}(n)}$ is defined as:

$$
\delta=\arg \max _{n}\left(y_{l}^{X}(n)\right)
$$

$$
y_{l}^{X}(n)=I F F T\left\{Y_{l}(k) e^{j 2 \pi k / N} X_{l}^{*}(k)\right\}
$$

\section{FractionAl FOURIER TRANSFORM}

Time and Frequency form two orthogonal axes in the time-frequency plane. As the conventional Fourier transform is to reformulate a defined signal either in the time domain as a function of frequency, it can be seen as a rotation of $\pi / 2$ in the trigonometric direction in the time-frequency plane. The fractional Fourier transform is intended as a generalization of the classic Fourier transform .It could be rotated to any angle $\alpha$ in the plane time-frequency. For each $\alpha$ value, FRFT is a different representation of the original signal function of time. So actually, the FRFT corresponds to a one parameter family of transformation. For $\alpha=0$, the FRFT coincides with the identity transformation which restores the original signal in the time domain. For $\alpha=\pi / 2$, it yields to the conventional Fourier transform. Mathematically, we defined the fractional 
International Journal of Wireless \& Mobile Networks (IJWMN) Vol. 8, No. 2, April 2016

Fourier transform a signal $\mathrm{s}(\mathrm{t})$ is defined as follows :

$$
F_{\alpha}(s)=S_{\alpha}(u)=\int_{-\infty}^{+\infty} s(t) K_{\alpha}(t, u) d t
$$

Where $\mathrm{p}$ is a real number known as FRFT order, $\alpha=\mathrm{p} \pi / 2$ is the angle of FRFT, and $\mathrm{K}_{\alpha}(\mathrm{t}, \mathrm{u})$ is the kernel of FRFT:

$$
= \begin{cases}\sqrt{\frac{1-j \cot (\alpha)}{2 \pi}} \exp \left(j \frac{t^{2}+u^{2}}{2} \cot (\alpha)-j u t c s c(\alpha)\right) & \alpha \neq n \pi \\ \delta(t-u) & \alpha=2 n \pi \\ \delta(t+u) & \alpha+\pi=2 n \pi\end{cases}
$$

Fractional Fourier transform is used in many field such in [12].

\section{Proposed Method And Simulation Results}

The performances of FRFT are shown in this section. For CFO estimation, we use the same technique done by Classen [3] with FRFT and CAZAC sequences inserted in comb-type. For STO estimation, we use Shi \& Serpedin method [11] but with the use of FRFT instead of FFT.

Table 3 shows the simulation parameters.

Table 3: The parameters for simulation

\begin{tabular}{|l|l|}
\hline Modulation & 16 QAM \\
\hline Number of sub-carrier & 128 \\
\hline Cyclic Prefix Length $(\mathrm{Ng})$ & 32 \\
\hline Pilot sequences & CAZAC sequences \\
\hline Number of Bits per Symbol & 4 \\
\hline Pilot Spacing (Nps) & 4 \\
\hline CFO & 0.5 \\
\hline STO & -3 \\
\hline Number of iteration & 100 \\
\hline Signal to Noise Ratio (SNR) range & $0: 30$ \\
\hline
\end{tabular}

It is clearly shown in figure 5 that the use of fractional Fourier Transform with Classen method gives better results in term of MSE compared to Classen method using conventional Fourier Transform and other known techniques (CP based and Moose technique).

It is also shown in figure 6 that the method done by Shi \& Serpedin with FRFT is more efficient than the one done with FFT. Graphs show the efficiency of using FRFT compared to the most known method (Schmidt and Cox). 
International Journal of Wireless \& Mobile Networks (IJWMN) Vol. 8, No. 2, April 2016

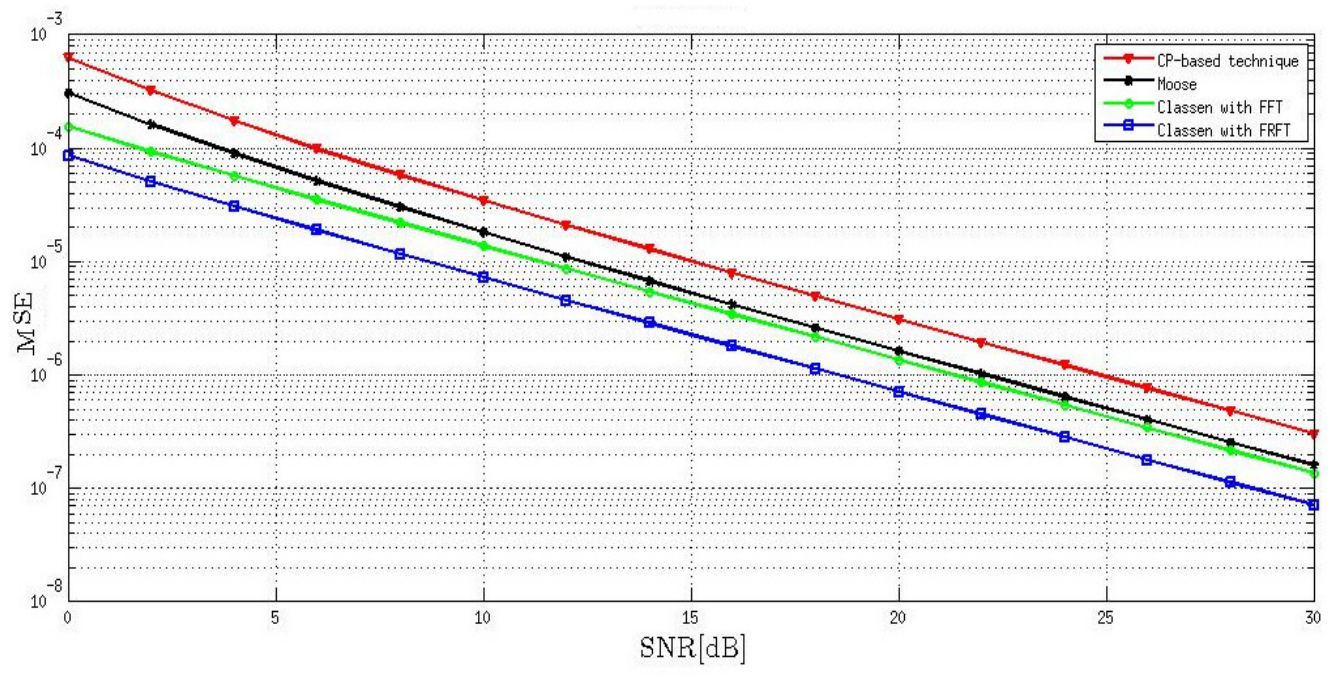

Figure 5: MSE of CFO of most known method (CP based, Moose method, Classen with FFT) and one done with FRFT.

To more explain the effect of fractional Fourier transform of CFO, we draw the graph for different order of FRFT, figure 6.

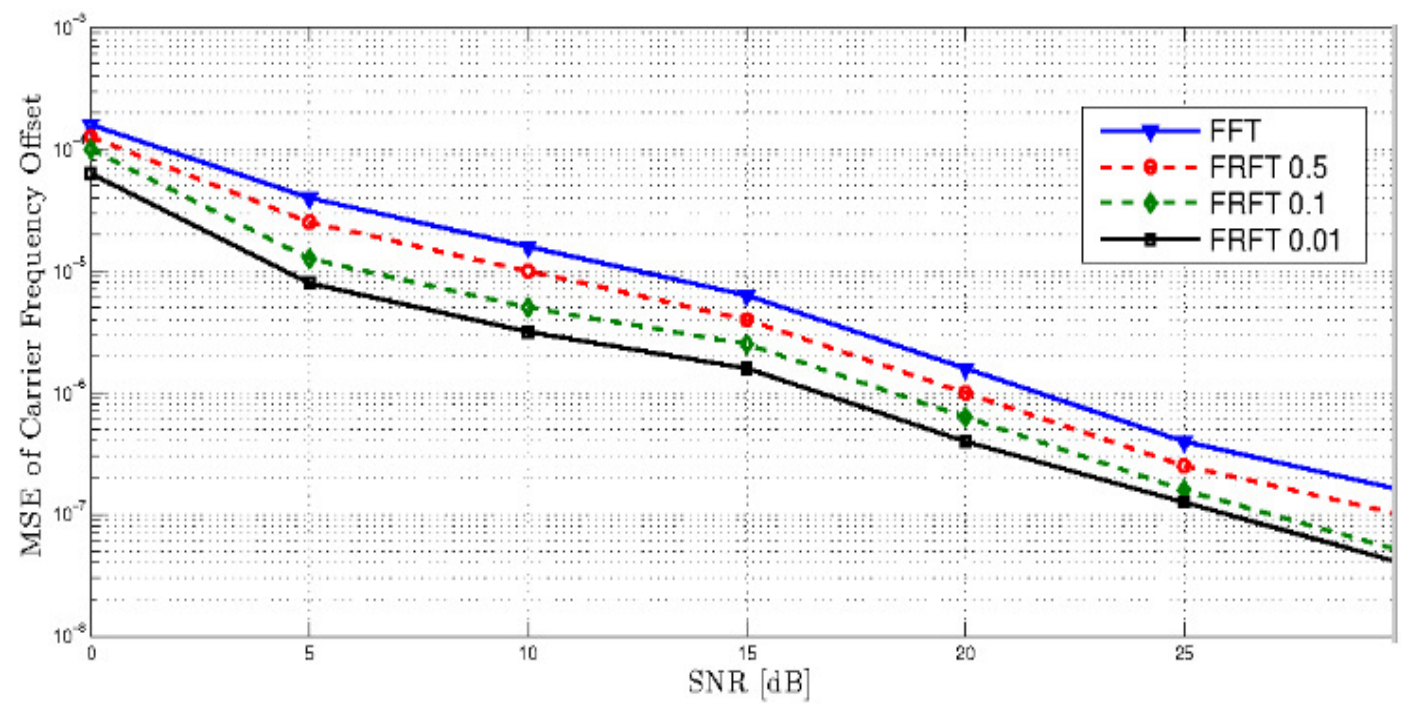

Figure 6: MSE of CFO for FFT and FRFT for different order.

Figure 6 shows the effect of FRFT order on MSE of carrier frequency offset. It shows that when the order becomes small (i.e. it tends to identity) the MSE becomes better and when the order is great (i.e. it tends to FFT) the results becomes worse. For example, when the order is equal to 0.01 and for SNR equal to $15 \mathrm{~dB}$, we obtain a gain equal to $6 \mathrm{~dB}$. 
International Journal of Wireless \& Mobile Networks (IJWMN) Vol. 8, No. 2, April 2016

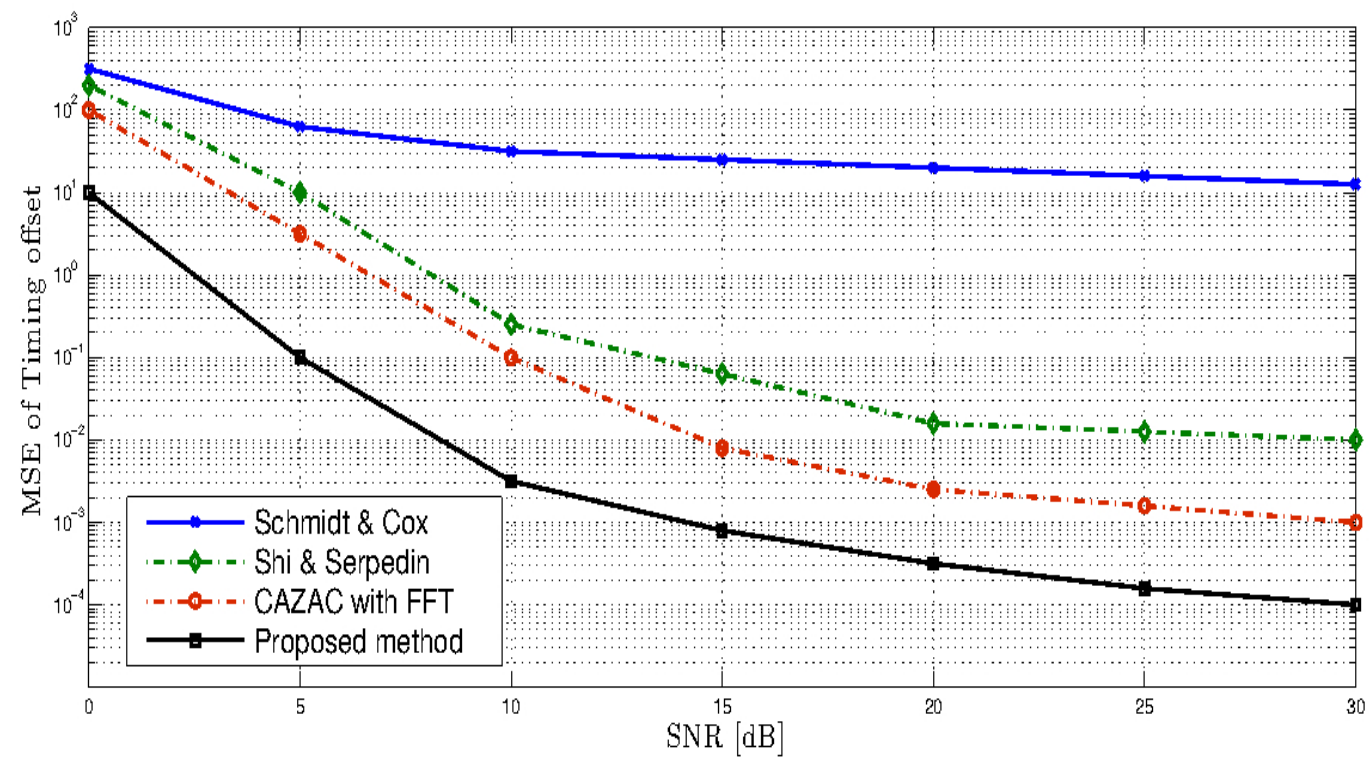

Figure 7: MSE of STO for Scmidt \& Cox, Shi \& Serpedin and Shi \& FFT with CAZAC \& FRFT with CAZAC sequences.

It is also shown in figure 7 that the method done by Shi \& Serpedin with FRFT is more efficient than the one done with FFT. Graphs show the effiency of using FRFT compared to the most known method (Schmidt and Cox).

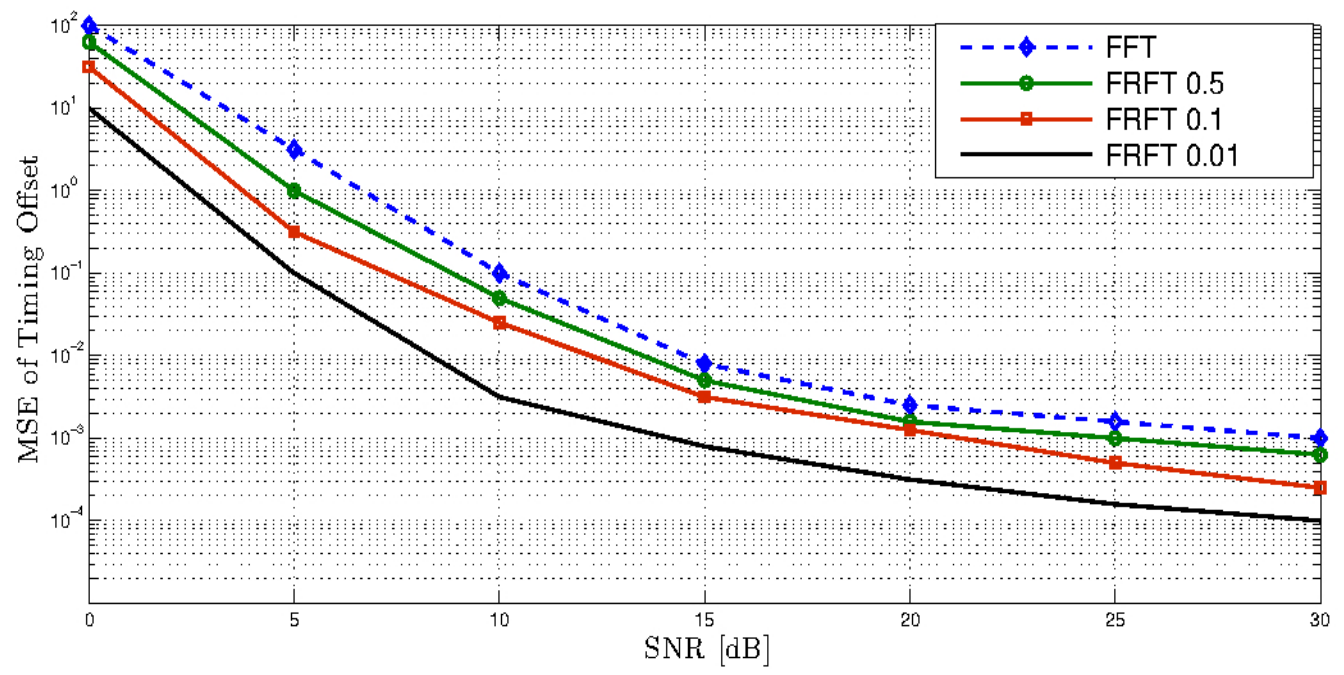

Figure 8: MSE of timing offset for FFT and FRFT for different order.

Figure 8 shows the effect of FRFT order on MSE of carrier frequency offset. It shows that when the order becomes small (i.e. it tends to identity) the MSE becomes better and when the order is great (i.e. it tends to FFT) the results becomes worse. For example, when the order is equal to 0.01 and for SNR equal to $15 \mathrm{~dB}$, we obtain a gain equal to $9 \mathrm{~dB}$. 
International Journal of Wireless \& Mobile Networks (IJWMN) Vol. 8, No. 2, April 2016

From these results, we conclude that FRFT is an efficient method for both CFO and STO estimation. For Further research in timing and frequency synchronization, we could use FRFT instead of FFT for better results.

\section{CONCLUSIONS}

This paper proposes a study for performance analysis for symbol timing offset (STO) and carrier frequency offset (CFO) estimation. This study compares Fractional Fourier transform to the conventional Fourier transform. The study uses most known methods for estimating CFO and STO with the use of FRFT and CAZAC sequences as pilot. The study demonstrates the efficiency of fractional Fourier transform. This study gives a good candidate (FRFT) for OFDM synchronization with the same complexity of conventional FFT.

\section{REFERENCES}

[1] Cho, Y.S., Kim J., Yang, W.Y. and Kang C. G., "MIMO-OFDM wireless communications with MATLAB", John Wiley and Sons, Asia, IEEE Press, 2010, pp.153-161.

[2] MOOSE P H, "A technique for orthogonal frequency division multiplexing frequency offset correction," IEEE Transactions on Communications. 1994, 42 (10), pp. 2908-2914.

[3] Classen, F. and Myer, H. "Frequency synchronization algorithm for OFDM systems suitable for communication over frequency selective fading channels," IEEE VTC94, pp. 1655-1659.

[4] Schmidl T M, Cox D C, "Robust frequency and timing synchronization for OFDM," IEEE Transactions on Communications. 1997, 45 (12), pp.1613-1621.

[5] Chun linYan, Shaoqian Li, Youxi Tang, Xiao Luo, and Jiayi Fang, "A Novel Frequency Offset Estimation Method for OFDM Systems with Large Estimation Range," IEEE Transactions on Broadcasting. 2006, 52(1), pp. 058-061.

[6] Wen-Jeng Lin; I-Cheng Liu; Jung-Shan Lin "Novel frequency offset estimation for IEEE 802.11p standard in V2V channels", ITS Telecommunications (ITST), 2012 12th International Conference on, On page(s):264 - 268.

[7] Tourtier, P.J., Monnier, R., and Lopez, P. "Multicarrier modem for digital HDTV terrestrial broadcasting" .Signal Process., 5(5), 379403, 1993.

[8] Speth,M., Classen, F., and Meyr,H. "Frame synchronization of OFDM systems in frequency selective fading channels". IEEE VTC97, pp.18071811, 1997.

[9] T. M. Schmidl and D. C. Cox , "Robust Frequency and Timing Synchronization for OFDM", IEEE Trans. on Commun. , pp.1613 -1621, 1997.

[10] D. C. Schmidl and D. C. Cox , "Low-overhead, low-complexity [burst] synchronization for OFDM" , IEEE Int. Conf. on Commun, vol. 3 ,pp.1301 -1306, 1996.

[11] K. Shi and B. E. Serpedin. "Coarse frame and carrier synchronization of OFDM systems: A new metric and comparison”, IEEE Trans. Wireless Commun., vol. 3, no. 4, pp. 12711284, Jul. 2004.

[12] Massimiliano (Max) Martone , "A Multicarrier System Based on the Fractional Fourier Transform for Time-Frequency-Selective," IEEETransactions on Communications, VOL. 49, NO. 6, June 2001.

[13] Yushi Shen and Ed Martinez, "Channel Estimation in OFDM Systems", Freescale Semiconductor, Inc., 2006.

[14] Coleri, S., Ergen, M., Puri, A., and Bahai, A.,"Channel Estimation Techniques Based on Pilot Arrangement in OFDM Systems," IEEE Transactions on Broadcasting, vol.48, pp.223229, Sept. 2002.

[15] Sinem Coleri, Mustafa Ergen,Anuj Puri, Ahmad Bahai, "A Study of Channel Estimation in OFDM Systems", IEEE 56th Vehicular technology conference.

[16] Minn, H. and Bhargava, V.K. (1999), An investigation into time-domain approach for OFDM channel estimation. IEEE Trans. on Broadcasting,45(4), 400409. 
International Journal of Wireless \& Mobile Networks (IJWMN) Vol. 8, No. 2, April 2016

Author

Cherif Rezgui is graduate astelecommunication Engineer from higher school communication (Sup'Com) in 2004, his master degree in 2011 from national school of engineers of Tunis. He is actually working on Ph.D. He is working in the field of MIMO, OFDM, Chanel estimation and synchronization. 